\title{
EVIDENCE FOR A GALACTIC COMPONENT OF THE DIFFUSE X-RAY BACKGROUND
}

\author{
B. A. COOKE, R. E. GRIFFITHS and K. A. POUNDS \\ $X$-ray Astronomy Group, Dept. of Physics, University of Leicester
}

\section{Introduction}

It is widely believed that the diffuse X-ray background, observed on several occasions over the energy range from $0.25 \mathrm{keV}$ to above $1 \mathrm{MeV}$ has an extragalactic origin. Evidence for this comes from the generally reported isotropy above several $\mathrm{keV}[1$, $2,3]$ and the observed galactic latitude dependence at $0.25 \mathrm{keV}$, believed to result from the interstellar attenuation of these low energy photons in passage through the Galaxy $[4,5]$.

The present paper reports some results from a recent high sensitivity sky survey in the 1.4-18 keV band, which show a clear excess of radiation in the galactic plane in the Vela-Carina-Centaurus region $\left(l^{\mathrm{II}}=220-320^{\circ}\right)$. No strong discrete X-ray sources are seen in this part of the Milky Way and the possibility of this flux representing a Galactic component of the diffuse background radiation is suggested. A study of the published results of previous rocket and balloon experiments has been made with the conclusion that the presently reported 'anistropy' could have remained unobserved in each case, for one or other of the following reasons. First, the great majority of observations have been concentrated on the strong source regions - in Scorpius, Sagittarius and Cygnus - where a narrow band of excess background radiation along the galactic equator would be obscured by the sources. Second, no other survey experiment of comparable sensitivity and resolution has searched the galactic longitudes covered in the present flight, where the absence of discrete sources is marked.

\section{Flight details}

The present observations were made from an unstabilised Skylark rocket, SL 723, launched from Woomera, South Australia, at $20^{\mathrm{h}} 00^{\mathrm{m}}$ local time on June 12, 1968. Two proportional counter detectors, each of $1385 \mathrm{~cm}^{2}$ effective area, were mounted viewing sideways from the nose-cone section of the rocket, detector $\mathrm{C} 1$ covering the energy range $1.4-3.6 \mathrm{keV}$ in 7 energy channels and detector $\mathrm{C} 2$ the range $2-18 \mathrm{keV}$ in 9 energy channels. The field of view of each detector was $28^{\circ}$ and $4^{\circ}$ (FWHM) with the greater collimator extension parallel to the longitudinal rocket axis for $\mathrm{C} 2$ and for six-tenths of $\mathrm{Cl}$, the remaining section of the $\mathrm{Cl}$ collimator being canted at $40^{\circ}$ to the major rocket axis. During the flight, the rocket spun at a constant rate of $75^{\circ}$ per second, while the spin axis precessed about a flat cone at $0.84^{\circ}$ per second. Attitude information was provided by on-board moon sensors and magnetometers, with an estimated rms error of $1.8^{\circ}$. 
During the early part of the flight the detectors scanned roughly along the galactic equator, covering the zone from $l=220^{\circ}$ to $l=320^{\circ}$ and then across the ScorpiusSagittarius region. These 'along scans' are shown as 1-16 in Figure 1. In the second half of the flight, part of the same low latitude region was scanned again in a perpendicular direction, these 'across scans' being 1'-20' in Figure 1.

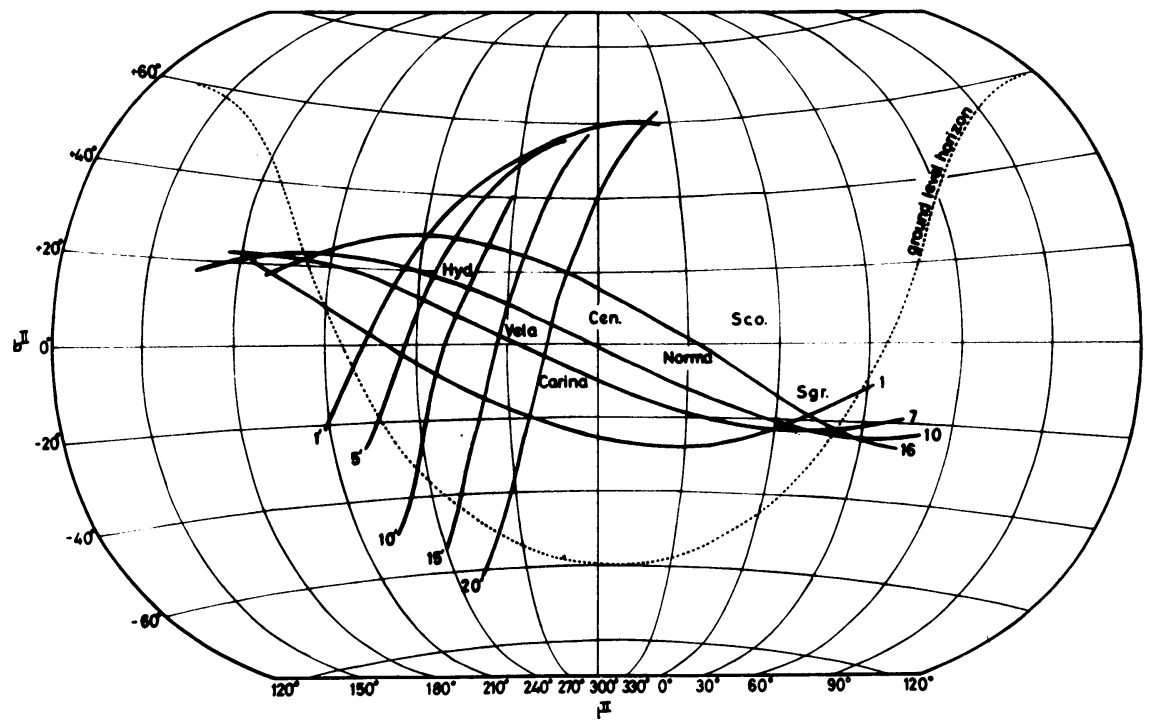

Fig. 1. Sky map in $l^{\mathrm{II}}, b^{\mathrm{II}}$ coordinates of the region of the galactic equator surveyed on June 12 , 1968 by Skylark rocket SL 723.

\section{Results}

The accumulated data from groups of scans across the galactic equator are plotted in Figure 2, where separate scans have been added by using the galactic equator crossing as a common time origin. The occurrence of a persistent peak at the position of the galactic equator is clear in every group of scans, covering the galactic longitudes from 220 to $285^{\circ}$. The small peak to the left of the equator position, seen on scans $13^{\prime}-20^{\prime}$ is due to a discrete source in the Canopus region, as yet unreported, but confirmed in separate scans across this area of sky.

Evidence that the galactic equator peak does not arise from a $f e w$ discrete sources is provided by the scans along the equator. Accumulated data from groups of these scans are shown in Figure 3. In the case of these almost parallel scans, the addition was carried out by summing each counting interval separated by a complete rocket spin period, the exactness of this procedure then being confirmed by plotting each scan on a globe. Scans 1-10 are plotted in Figure 3a, from which it may be seen that the X-ray count lies on the all-sky, isotropic background level in the Puppis region, while still North of the galactic equator, and then increases significantly as the equator is reached in the region of Vela. No strong source is seen in the position of Vel XR-1, 


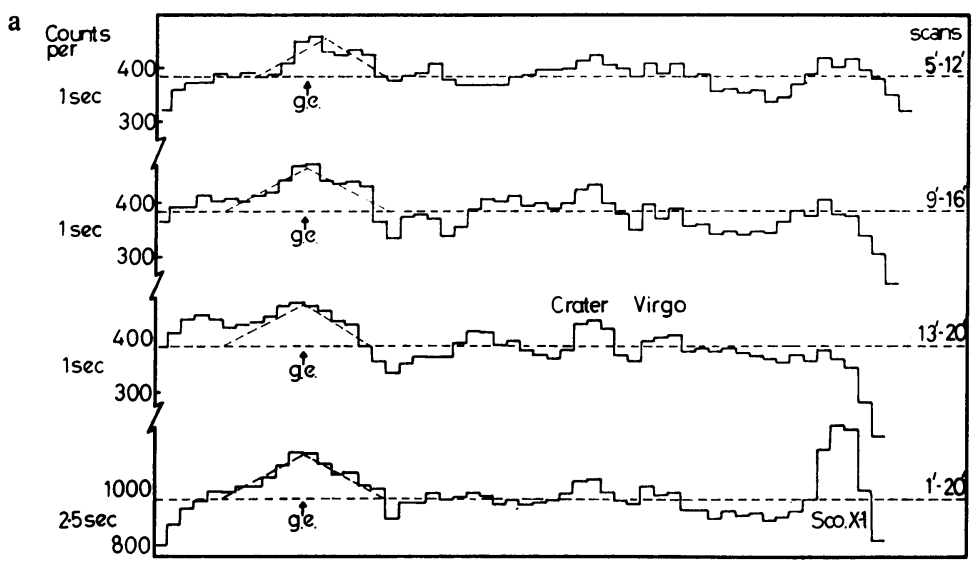

SCANS ACROSS GALACTIC EQUATOR 2-18 KeV

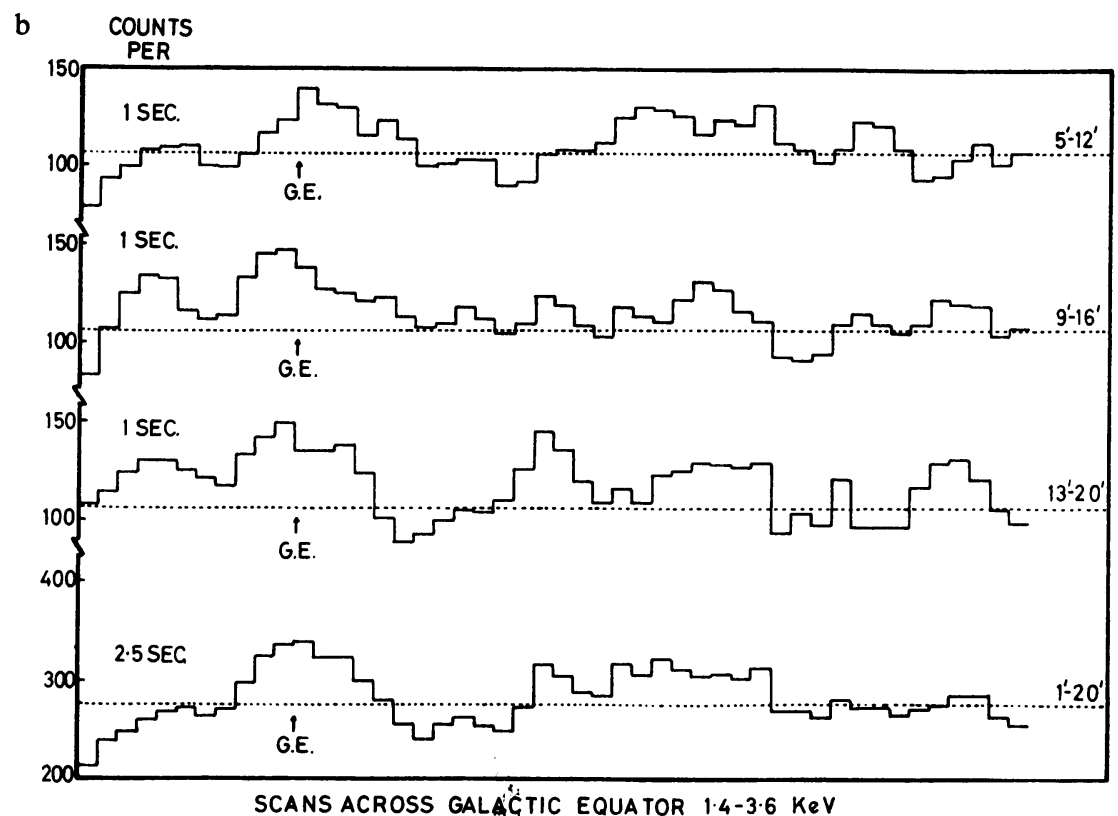

Figs. 2. Evidence for the X-ray enhancement at the position of the galactic equator produced by summation of groups of scans for detector $\mathrm{C1}(1.4-3.6 \mathrm{keV})$ and $\mathrm{C} 2(2-18 \mathrm{keV})$.

the maximum intensity allowed by the present data being 0.15 photon $\mathrm{cm}^{-2} \mathrm{sec}^{-1}$ above $2 \mathrm{keV}$. This is significantly less than the A.S. and E. Group's measurement [6], obtained in February 1968 and it seems probable, therefore, that Vel XR-1 is a variable or short-lived emitter. The estimated count rate profile that would have been seen by the present instrument from a source of the A.S. and E. strength is shown in Figure 3a. Further along these scans, there is some evidence for a source in the Centaurus region, which may be that previously reported as Cen XR-3 [7]. 
a

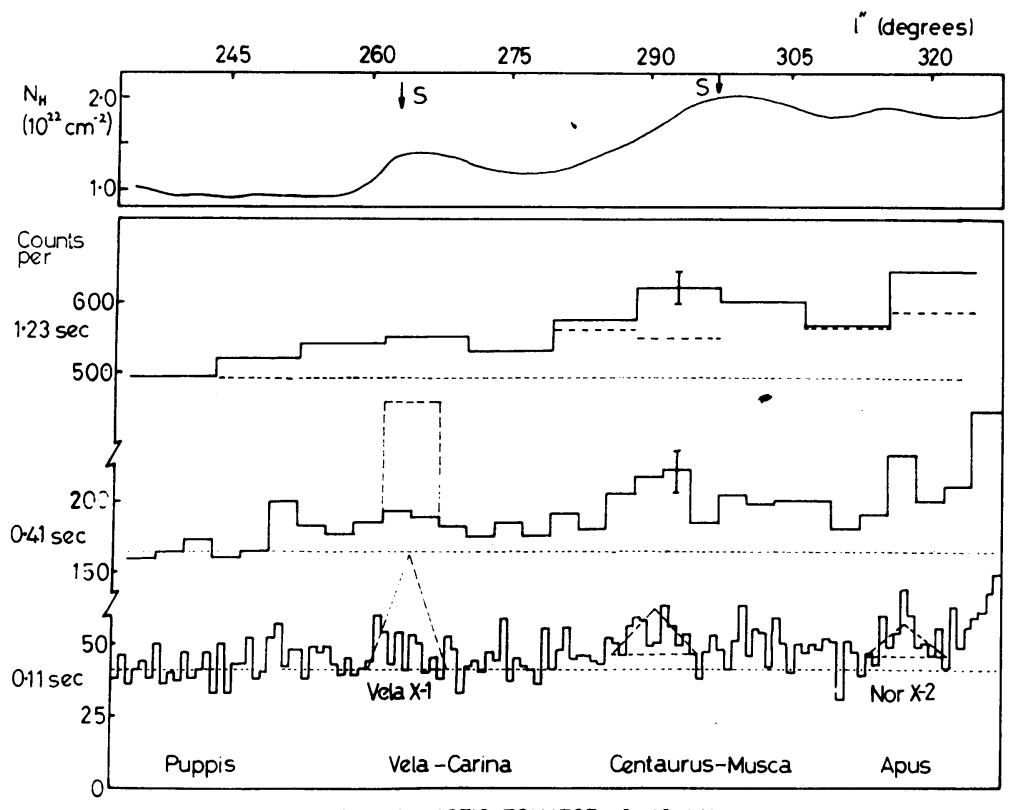

SCANS ALONG GALACTIC EQUATOR 2-16 KeV

b

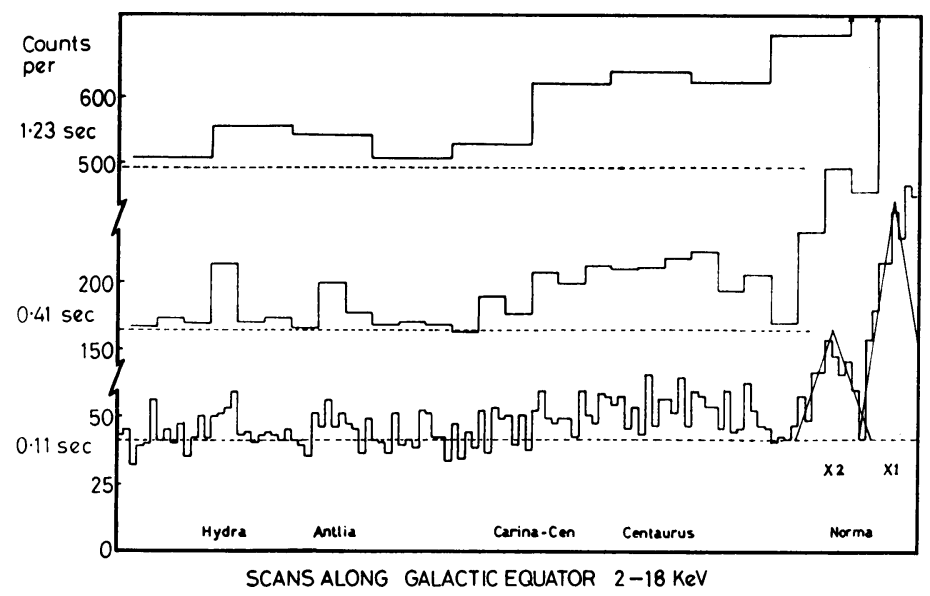

Figs. 3. Summed groups of scans along the galactic equator showing how the X-ray enhancement varies with galactic longitude in the Vela-Carina region (3a) and the Carina-Centaurus region (3b). The dashed triangle at Vela X-1 gives the strength measured by AS and E [6]. Those at Cen X-3 and Nor $\mathrm{X} 1$ and $\mathrm{X} 2$ give the strengths measured in this flight.

The measured intensity above $2 \mathrm{keV}$ is 0.25 photon $\mathrm{cm}^{-2} \mathrm{sec}^{-1}$, at a position $10^{\mathrm{h}} 15^{\mathrm{m}}$ right ascension and $-74^{\circ}$ declination, some $10^{\circ}$ from the location of Cen XR-3 reported by the LRL Group. Of particular interest is the non-appearance of Cen XR-2 on our data. This source, which in April 1967 was stronger [3] than Sco X-1 faded below a level of 0.6 photon $\mathrm{cm}^{-2} \mathrm{sec}^{-1}$ in September of that year [8]. The upper limit in June 1968, from the present data, is a factor of 4 lower still. Recently, 
however, the provisional results from two rocket launches from India in November, 1968 have been reported [9], indicating that at this time Cen XR-2 has re-appeared at a strength of about 1.0 photon $\mathrm{cm}^{-2} \mathrm{sec}^{-1}$, above $2 \mathrm{keV}$. Observations above $20 \mathrm{keV}$ from balloons by the MIT group gave positive measurements in October 1967, at a level [10] roughly consistent with the LRL upper limit at lower energies. A preliminary report [11] of a new MIT balloon flight in April 1969, however, reports no evidence of Cen XR-2 to a level of one-third of the October 1967 flux! Beyond the Centaurus-Musca region, the scans plotted in Figure 3a pass South of Norma, a partial view of the NOR XR-2 source being obtained, and then the count rate rises steeply with the detection of the strong NOR XR-2 and ARA sources. It is perhaps important to record that the Norma, Ara, Scorpius and Sagittarius sources are all clearly seen in the continuation of these same scans, with intensities and positions close to those reported from previous flights by the NRL [12], A.S. and E. [13] and MIT [14] Groups. This gives us confidence in the reliability of the non-observation of several sources from the present flight, as discussed above. The spectra and intensities of the galactic sources from Norma to Sagittarius will be presented in a separate paper.

A re-examination of Figure $3 a$ can now be made, allowing for the flux due to the observed discrete sources in Centaurus and Norma. This is done (short dashed lines) in the third panel up of Figure 3a. A gradual increase in the excess above the isotropic background level is now evident as the scans progress from galactic longitude $245-320^{\circ}$, in general conformity with the neutral hydrogen column density, shown in the top panel. Reference to Figure $3 \mathrm{~b}$ confirms this trend. Here are plotted the counts accumulated in the 'along equator' scans 7-16 and reference to Figure 1 shows that these pass through Hydra and Antlia before converging on the galactic equator near longitude $280^{\circ}$, in the region of Carina-Centaurus. Once again, the count rate is seen to rise markedly from the isotropic background level when the galactic equator enters the field of view. These scans cross directly over the Norma sources which are shown at their full relative intensities in Figure $3 \mathrm{~b}$.

Thus, we observe a clear excess of X-rays from the galactic equator region whenever this is viewed. The width of the emission band can be derived from the across-scan data of Figure 2, to be $6^{\circ}$ or less and its location indicates it is certainly of galactic origin, being either unresolved sources or a diffuse galactic flux, or both. The galactic excess near $l=260^{\circ}$ can be expressed as 0.3 photon $\mathrm{cm}^{-2} \mathrm{sec}^{-1} \mathrm{rad}^{-1}$, rising to 0.5 photon $\mathrm{cm}^{-2} \mathrm{sec}^{-1} \mathrm{rad}^{-1}$, in the Centaurus region, near $l=300^{\circ}$. Further towards the galactic centre it becomes very difficult to deduce the intersource background flux, since the Ara, Scorpius and Sagittarius sources are strong and numerous. A minimum count rate obtained near $l=350^{\circ}$ does not exclude a further factor of 2 increase in this galactic component, that is, to 1 photon $\mathrm{cm}^{-2} \mathrm{sec}^{-1} \mathrm{rad}^{-1}$.

\section{Discussion}

It may be that the galactic equator excess is due to a number of unresolved sources 
which lie below the sensitivity threshold of the present survey and in this regard it is interesting to compare the rquired source distribution with the known galactic sources. It has been suggested [15] that the Scorpius-Sagittarius source may be 'typical' galactic sources, yielding a flux at earth's distance of between 1 and 2 photons $\mathrm{cm}^{-2}$ $\mathrm{sec}^{-1}$ above $2 \mathrm{keV}$ and also that their most probable location is in the Sagittarius spiral arm, at a mean distance of 2 or 3 kiloparsecs. Again, the Cygnus group of sources [13], probably located in one extension of the local spiral arm, would lie at a similar, and reasonable, distance if they are of the same intrinsic strength. If it is accepted that the local arm extends in the opposite direction through Vela [16], then a very different situation must exist in this extension. The presently reported flux and the smooth distribution of excess counts as shown in Figure 3 require at least 10 sources to be spread out over the Vela-Centaurus region of the Milky Way. The average flux at the earth from each source is then only 0.04 photon $\mathrm{cm}^{-2} \mathrm{sec}^{-1}$, representing a 'typical' galactic source at an unreasonable large distance of 10 to $15 \mathrm{kpc}$. Of course, it may be that the Vela-Centaurus regions contain a much weaker class of galactic X-ray source. Higher resolution and more sensitive studies, using stabilised rockets, will be required to pursue this possibility.

An alternative explanation, considered briefly below, lies in the possibility that the low latitude enhancement is due mainly to a diffuse galactic X-ray flux, connected with the concentration of matter, radiation or cosmic rays in the galactic plane. The gradual increase of the excess X-radiation towards the galactic centre, in conformity with the increasing neutral hydrogen $[17,18]$ column density, is shown in Figure $3 a$. The spectral distribution of the enhancement is shown in Figure 4 and may be fitted with a power law, similar to the isotropic background spectrum [19] perhaps indicating a similar production mechanism. Several possible processes that could contribute towards a significant diffuse galactic X-radiation are briefly discussed below.

(A) Suprathermal proton bremsstrahlung, arising from the collisions of 2-20 MeV cosmic ray protons with ambient electrons in the spiral arms, has been considered [20] as a source of 1-10 keV X-rays. This flux will exhibit the (probable) power law spectrum of the protons and will be correlated in intensity with the neutral hydrogen. However, the heating of interstellar H I regions [21] places a limit on the proton flux which, in conjunction with the neutral hydrogen column densities shown in Figure 3a, yield an X-ray flux from the galactic plane more than an order of magnitude less than that observed here. Stecker has recently [22] presented evidence, however, that the interstellar gas density may be up to an order of magnitude higher than that estimated from $21 \mathrm{~cm}$ emission.

(B) Electron capture by heavy cosmic ray nuclei, colliding with the neutral hydrogen in the galactic disc, with the subsequent radiative cascade of the excited ions, yielding $\mathrm{X}$-ray line emission. This process has recently been examined by Silk and Steigman [23], who show that it may yield fluxes comparable to the isotropic background $\mathrm{X}$-radiation, in the 1-10 keV band. The strongest predicted lines, silicon and magnesium Ly- $\alpha$, are reproduced from the above paper in Figure 4, Doppler broadening 
spreading the emission over the energy band 1-2.5 keV. The crude spectral data of the galactic X-ray enhancement does allow some steepening in the spectrum below $2.5 \mathrm{keV}$, but the statistical significance of the low energy points is clearly insufficient to prove the existence of this flux. It does seem, however, that the measured spectrum above 3 or $4 \mathrm{keV}$ requires a different source mechanism, unless there is a much larger abundance of the heavier elements $(\mathrm{P}-\mathrm{Ni})$ in the few $\mathrm{MeV}$ range than is observed at higher energies [24].

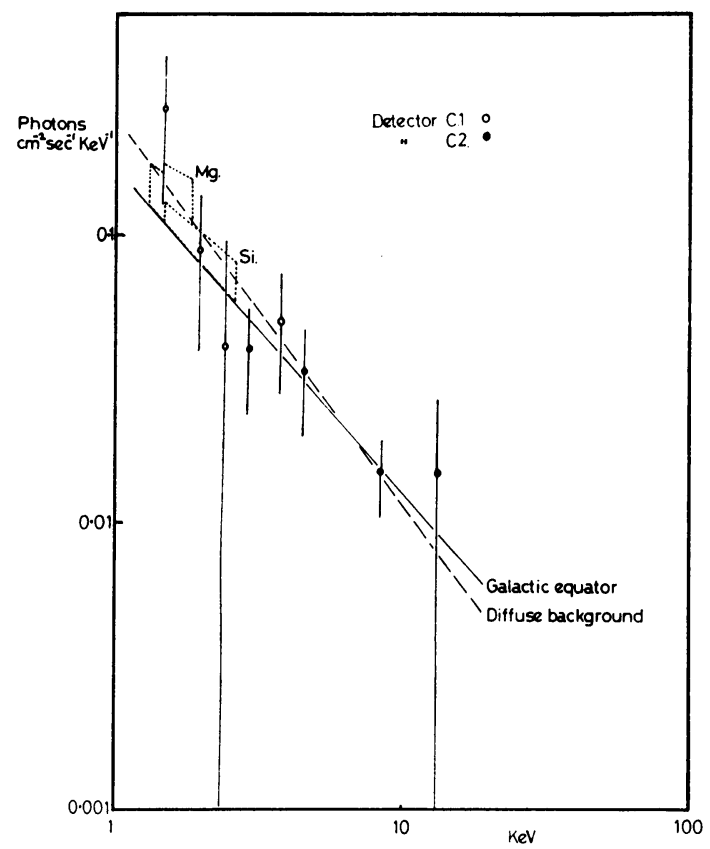

Fig. 4. The X-ray spectrum of the galactic equator enhancement compared with that of the diffuse $\mathrm{X}$-ray background. The blocks labelled $\mathrm{Mg}$ and $\mathrm{Si}$ represent the line strength due to those elements from the paper of Silk and Steigman [23].

(C) Inverse Compton radiation. It is generally assumed that the most likely source of the isotropic diffuse X-radiation is by inverse Compton scattering of high energy electrons in the distant reaches of the universe, and the contributions from both dense galaxies and quasars [25] and the 'universal' black-body (3K) radiation [26] have been estimated. The latter radiation falls short of explaining the present galactic excess by over an order of magnitude, however, and it is generally accepted that the flux of the appropriate $\mathrm{GeV}$ electrons in interstellar space is reasonably well known [27], since solar modulation is weak at these electron energies.

The Compton X-ray production could be raised to the observed intensity if the strong far infra-red radiation reported by Shivanandan et al. [28] is confirmed and pervades the whole galaxy. However, the existence of a widespread infra-red back- 
ground of this intensity is hard to reconcile with the observed excitation of interstellar molecules [29].

Finally, there remains the possibility of inverse Compton scattering of starlight $(\mathrm{T} \sim 6000 \mathrm{~K})$ in the galactic disc. Rees and Silk [30] have recently put forward an explanation of the $100 \mathrm{MeV} \gamma$-ray flux, observed as an apparently diffuse, narrow band of radiation from the Milky Way [31, 32, 33, 34], in which it is proposed that the $\gamma$-rays arise as bremsstrahlung from the collisions of $100 \mathrm{MeV}$ electrons with the interstellar gas atoms. The essential point of the Rees and Silk paper is that the required large flux of $100 \mathrm{MeV}$ electrons does not contradict any available observational data. It is of particular interest here to note that an extension of their assumed electron spectrum from $100 \mathrm{MeV}$ to $15 \mathrm{MeV}$ would produce an excess of 2-10 keV X-rays in the galactic plane, by inverse Compton interactions with starlight, comparable with that indicated in the Vela-Centaurus region in the present paper. Assuming a starlight energy density of $0.5 \mathrm{eV} \mathrm{cm}^{-3}$, and allowing for a factor of 2 increase in the scattering efficiency [35] due to the anisotropic starlight flux (travelling mainly in the galactic disc and directed outward from the centre), the energy density of the $\sim 20 \mathrm{MeV}$ electrons required is $0.25 \mathrm{eV} \mathrm{cm}^{-3}$, to yield the 'observed' diffuse galactic X-radiation. It is noteworthy that this large electron flux would provide a heat input to the interstellar medium of the same order as that assumed by Field et al. [36], these authors attributing the heating to a low energy proton component, for which there is less direct evidence. Rees and Silk (private communication) also point out that the expected turn-over in the electron spectrum, at about $10 \mathrm{MeV}$, due to ionisation losses, could in addition explain the intensity and spectrum of $1-10 \mathrm{MeV}$ $\gamma$-rays recently observed by Vette et al. [37].

\section{Reference}

[1] Gould, R. J.: 1967, Am. J. Phys 35, 376.

[2] Gorenstein, P., Kellogg, E. M., and Gursky, H.: 1969, Astrophys. J. 156, 315.

[3] Cooke, B. A., Pounds, K. A., Stewardson, E. A., and Adams D. J.: 1967, Astrophys. J. 150, L189.

[4] Bowyer, C. A., Field, G. B., and Mack, J. E.: 1968, Nature 217, 32.

[5] Kraushaar, W. L.: 1970, this volume.

[6] Gursky, H. Kellogg, E. M., and Gorenstein, P.: 1969, Astrophys. J. 154, L71.

[7] Chodil, G., Mark, H., Rodrigues, R., Seward, F., Swift, C. D., Hiltner, W. A., Wallerstein, G., and Mannery, E. J.: 1967, Phys. Rev. Letters 19, 681.

[8] Chodil, G., Mark, H., Rodrigues, R., and Swift, C. D.: 1968, Astrophys. J. 152, L45.

[9] Rao, U. R., Chitnis, E. V., Prakasarao, A. S., and Jayanthi, U. B.: 1970, this volume p. 88.

[10] Lewin, W. H. G., Clark, G. W., and Smith, W. B.: 1968, Astrophys. J. 152, L49.

[11] Lewin, W. H. G.: 1970, this volume p. 144.

[12] Friedman, H., Byram, E. T., and Chubb, T. A.: 1967, Science 150, 374.

[13] Gursky, H., Gorenstein, P., and Giacconi, R.: 1967, Astrophys. J. 150, 275.

[14] Bradt, H., Naranan, S., Rappaport, S., and Spada, G.: 1968, Astrophys. J. $152,1005$.

[15] Giacconi, R., Gursky, H., and Van Speybroeck, L. P.: 1968, Ann. Rev. Astron. Astrophys. 6, 373.

[16] 'Review of Galactic Structure', in Landolt-Börnstein - Group VI 1, 623. (ed. by H. Voigt), Springer-Verlag, Berlin, 1965.

[17] Kerr, F. J.: 1967, Radio Astronomy and the Galactic System (ed. by H. van Woerden), Academic Press, p. 239.

[18] Schmidt-Kaler Th.: Radio Astronomy and the Galactic System (ed. by H. van Woerden), Academic Press, p. 171. 
[19] Seward, F., Chodil, G., Mark, H., Swift, C., and Toor, A.: 1967, Astrophys. J. 150, 845.

[20] Boldt, E. and Serlemitsos, P.: 1968, NASA/GSFC report X-611-68-385.

[21] Pikel'ner, S. B.: 1968, Soviet Astron. 11, 737.

[22] Stecker, F. W.: 1969, Nature 222, 865.

[23] Silk, J. and Steigman, G.: 1970, this volume p. 385.

[24] Comstock, C. M., Fan, C. Y., and Simpson, J. A.: 1969, Astrophys. J. 155, 609.

[25] Rees, M. J. and Setti, G.: 1968, Nature 219, 127.

[26] Brecker, K. and Morrison, P.: 1967, Astrophys. J. 150, L61.

[27] Webber, W. R.: 1968, Australian J. Phys. 21, 845.

[28] Shivanandan, K., Hauck, J. R., and Harwit, M. O.: 1968, Phys. Rev. Letters 21, 1460.

[29] Bortolot, N. J., Clauser, J. F., and Thaddeus, P.: 1969, Phys. Rev. Letters 22, 307.

[30] Rees, M. J. and Silk, J.: 1969, Astron. Astrophys 3, 452.

[31] Clark, G. W., Garmire, G. P., and Kraushaar, W. L.: 1968, Astrophys. J. 153, L203.

[32] Hutchinson, G. W., Ramsden, D., and Wills, R. D.: 1970, this volume p. 300.

[33] Sood, R. K.: 1969, Nature 222, 650.

[34] Valdez, J. V. and Waddington, C. J.: 1969, Astrophys. J. 156, L85.

[35] Baylis, W. E., Schmid, W. N., and Lüscher, E.: 1967, Z. Astrophys. 66271.

[36] Bowyer, C. S., Field G. B., and Mack J. E.: 1968, Nature 217, 32.

[37] Vette, J., Matteson, J. L., Gruber, D., and Peterson, L. E.: 1970, this volume p. 335. 